\title{
Effects of a Metal Plane on a Meandered Slot Antenna for UHF RFID Applications
}

\author{
Jikwon $\mathrm{Kim}^{1} \cdot \mathrm{Il}$-Young $\mathrm{Oh}^{1} \cdot$ Tae-Wan $\mathrm{Koo}^{1} \cdot$ Jun Chul $\mathrm{Kim}^{2} \cdot$ \\ Dongsu $\mathrm{Kim}^{2} \cdot$ Jong-Gwan Yook
}

\begin{abstract}
In this paper, the effects of a metal plane on the performance of a meandered slot RFID antenna are evaluated in a real environment, and 3 metal plane cases are considered (the most likely scenarios in which metal conductive materials are placed near the tag antenna). The metal plane effects can be categorized as matching degradation and antenna gain variation. First, matching degradation due to the antenna's induced mutual impedance is experimentally investigated. In addition, the gain variation is investigated to figure out the change in the radiation characteristics. With the derived antenna parameters, the read range is calculated with the Friis transmission equation and measured to analyze the effects of a metal plane on RFID system performance. The calculated and measured read range varies from $9.3 \mathrm{~m}$ to $19.1 \mathrm{~m}$ as the distance between the RFID antenna and the metal plane changes.
\end{abstract}

Key words: RFID, Mutual Impedance, Metal Effects, Friis Transmission Equation, Slot Antenna.

\section{I . Introduction}

RFID (Radio Frequency Identification) has gained tremendous attention due to the increasing industrial applications. Several types of RFID frequency bandwidths are categorized according to their use: LF (Low Frequency: $125 \mathrm{kHz}$ ), HF (High Frequency: $13.56 \mathrm{MHz}$ ), UHF (Ultra High Frequency: 860 960 MHz), and 2.45 GHz. Among these frequency bands, the UHF RFID systems are popular due to the realizable size of the antenna and far-field operation ranging from several meters to more than 10 meters. Each country has different frequency standards for UHF RFID applications; for example, $908 \sim 916 \mathrm{MHz}$ has been established as the industrial standard in Korea.

In overall passive RFID systems, the tag antenna is a critical component that determines the RFID reading range performance. The tag antenna collects power from the reader to operate the tag chip since it does not include an internal power source; thus, the UHF RFID systems cannot operate properly or even be turned on at all if the tag antenna is not functioning properly [1]. In most cases, the output impedance of a tag chip reveals capacitive characteristics: low output resistance and large negative output reactance. This is why UHF RFID antennas should have inductive impedance to provide complex conjugate matching for maximum power transfer between the antenna and the chip [2], [3].

Many techniques have been proposed to realize low resistance and highly inductive reactance with non-patch type antennas. A conventional method of realizing this condition is the T-matching section methodology that can be used as an impedance transformer [4] [6]. Another technique is an inductively coupled feed methodology that uses inductive coupling between the feed loop and the radiating body [7] [9]. These techniques can be implemented directly with any radiation structure at the resonance. In addition, slot techniques reveal complementary properties compared to a conductive antenna. Matching by slot modification with a genetic algorithm has been proposed in [10], and impedance manipulation with a conductive area is proposed in [11].

RFID tag antennas, however, suffer performance degradation in a real environment, since they are designed based on the assumption they will operate in free space. In practice, there are numerous foreign materials adja-

Manuscript received May 2, 2012 ; Revised May 30, 2012 ; Accepted June 5, 2012. (ID No. 20120502-012J)

${ }^{1}$ Dept. of Electrical and Electronics Engineering, Yonsei University, Seoul, Korea

${ }^{2}$ System Packaging Center of Korea Electronics Technology Institute, Seongnam, Korea.

Corresponding Author : Jong-Gwan Yook (e-mail : jgyook@yonsei.ac.kr)

This is an Open-Access article distributed under the terms of the Creative Commons Attribution Non-Commercial License (http://creativecommons.org/licenses/ by-nc/3.0) which permits unrestricted non-commercial use, distribution, and reproduction in any medium, provided the original work is properly cited. 
cent to tags, and metallic materials in particular significantly affect tag performance. The topic of this paper is the effect of a metal plane on an RFID meandered slot antenna. Metal effects on antennas can be categorized in two ways. The first is the induced mutual impedance that could degrade conjugate matching between the tag chip and the antenna. Various studies [12] [16] on matching degradation have focused mainly on very near distance operations, such as a few $\mathrm{mm}$ from the metal to the tag. However, mutual impedance is generally induced further than a few $\mathrm{cm}$ and even more than a halfwave length depending on the orientation and polarizations. In addition, most studies have focused on the bandwidth; very few works have paid attention to the impedance variation profiles [17]. The second is the antenna gain variation that can be modeled with the twoarray antenna system based on the image theory. A few studies on the gain variation [13], [18] have considered short distances and only one orientation of the tag antenna and the metal plane.

In this paper, the effects of an electrically large metal plane to the RFID meandered slot antenna are quantitatively investigated. This paper is organized with 4 sec-

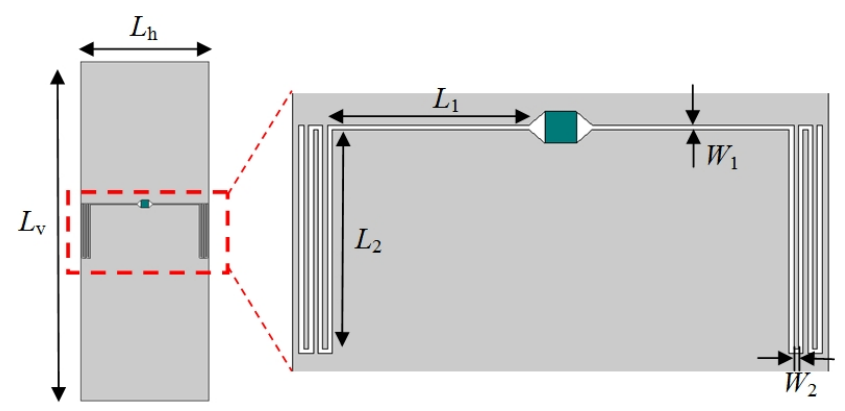

Fig. 1. The schematic of the meandered slot antenna and zoomed slot section [11].

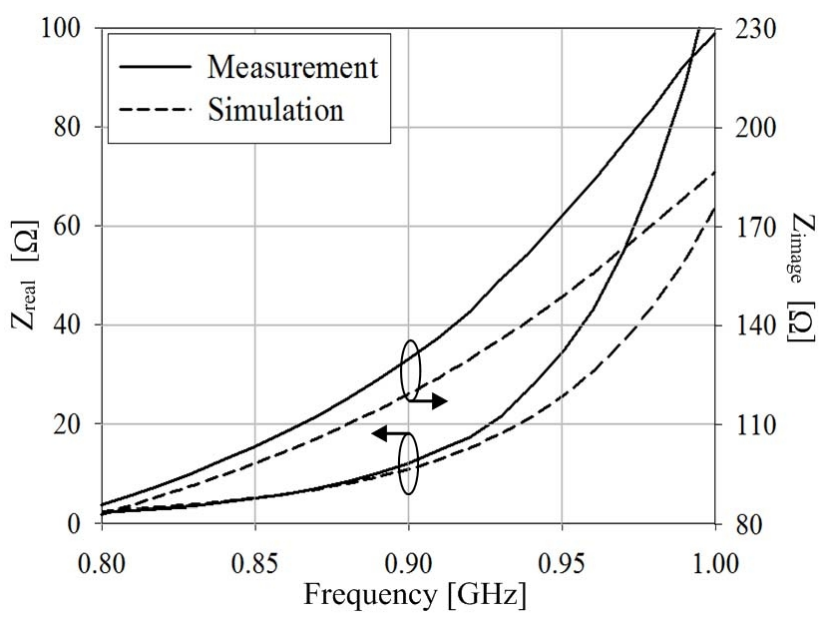

Fig. 2. The simulated and measured impedance response of the AUT (Antenna Under Test). tions. In section II, the designed meandered slot UHF RFID antenna with a center frequency of $912 \mathrm{MHz}$ is described. After the antenna is described, the matching degradation due to the induced mutual impedance and the gain are discussed in section III as a function of the antenna parameter variation. To quantify the performance variation of an RFID system, the read range as a figure of merit is discussed in section $\mathrm{IV}$ with the derived parameter variations from section III.

\section{II . AUT Characteristics}

In this work, a meandered slot dipole antenna is chosen for the test antenna based on its inductive impedance characteristics, which can be explained with the complementary relation of Babinet's principle and Booker's relationship. Since conventional dipole antennas have capacitive impedance near the resonance frequency owing to their open-circuited configuration, the complementary structure of the dipole antenna can realize inductive impedance.

The impedance of a slot antenna can be controlled with equation (1) without implementing additional matching networks. $Z_{c o n}$ is the impedance of the complementary conductive antenna, and $\eta$ is the intrinsic impedance in the free space. Babinet's principle and Booker's relationship are used to derive the slot impedance equation (1) under the assumption that there is an infinite metal area. Thus, the slot antenna can be matched to the complex conjugated impedance of an arbitrary CMOS (Complementary Metal-Oxide Semiconductor) tag chip by adjusting the length of the slot and the area of the metal area.

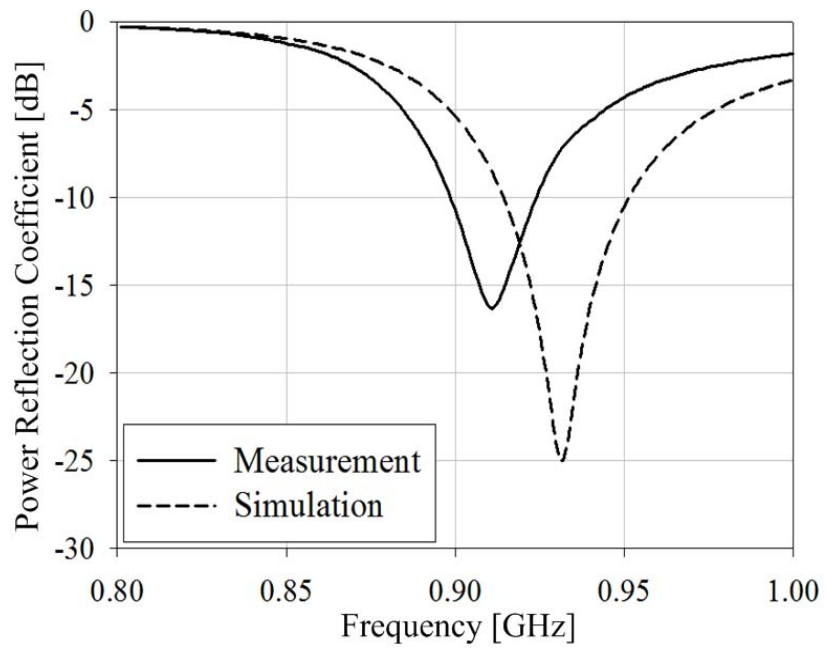

Fig. 3. The measured and simulated power reflection coefficient of the AUT. 


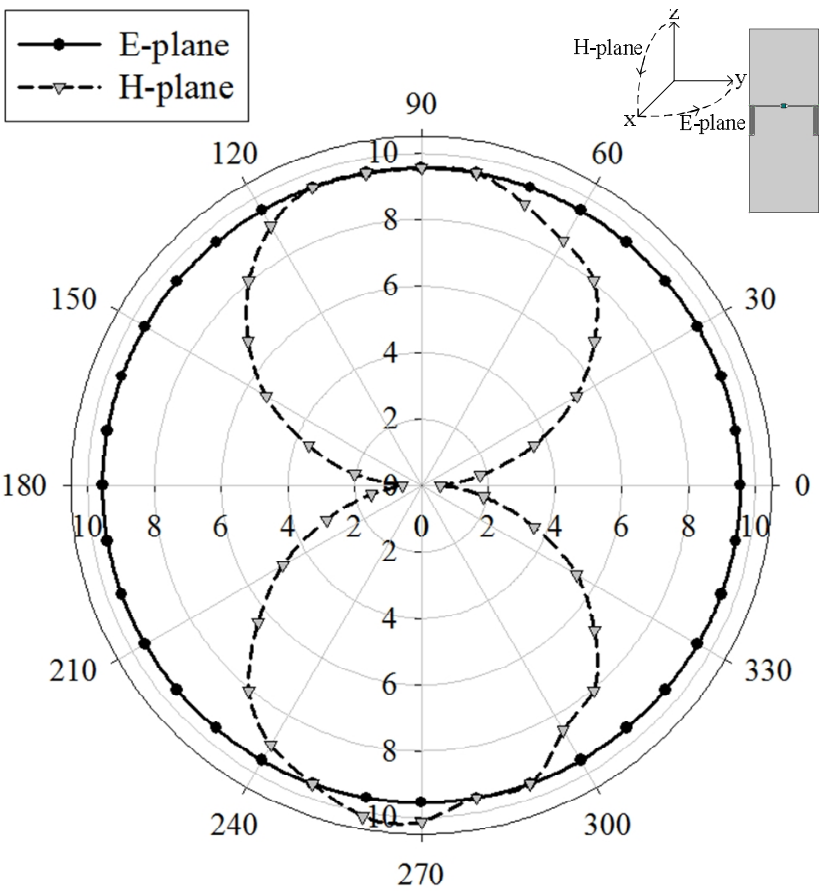

Fig. 4. Measured read range of AUT in the anechoic chamber.

$$
Z_{\text {slot }}=\frac{\eta^{2}}{2 Z_{\text {con }}}
$$

Fig. 1 shows the designed slot antenna; note that the slot dipole section is meandered to reduce the size of the overall slot length. The metal area (the grey area in the figure) is further extended to implement the required impedance value, a complex conjugate to the impedance of a Higgs-3 chip [11]. Since the impedance of the chip is $20-j 135 \Omega$ at $912 \mathrm{MHz}$, the AUT (Antenna under Test) is designed to have an impedance of $20+j 135 \Omega$ at the operating frequency.

The AUT is fabricated on a flexible substrate with $\varepsilon_{r}$ $=2.8$ and $\tan (\delta)=0.008$, and a substrate thickness of $0.028 \mathrm{~mm}$. The design parameters are $L_{1}=12.5 \mathrm{~mm}, L_{2}$ $=14.5 \mathrm{~mm}, W_{1}=W_{2}=0.3 \mathrm{~mm}, L_{v}=90 \mathrm{~mm}$, and $L_{h}=34 \mathrm{~mm}$ [11]. Fig. 3 shows the measured input impedance in free space. The input reactance increases inductively as expected from Babinet's principle, and the input resistance also increases significantly near the resonance frequency. Fig. 3 shows the return loss response, revealing excellent conjugate matching between the antenna and the tag chip. As a result, the measured 3-dB bandwidth is $80 \mathrm{MHz}$ with about $9 \%$ FBW (Fractional Band Width). The radiation pattern of the slot antenna is similar to that of a conventional short dipole with a simulated gain of $2.17 \mathrm{dBi}$. The measured read range in an anechoic chamber is shown in Fig. 4 with the omni-directional read range pattern in the E-plane. In the $\mathrm{H}$ - plane, the read range pattern reveals null point due to the mini- mum radiation of the antenna itself.

\section{Antenna Parameter Variation}

\section{3-1 Experimental Setup}

To explore the effect of an adjacent metal plane, the input impedance variation is measured as a function of distance, which is chosen every $1 \mathrm{~cm}$ from the metal plane. In addition, the gain variation is calculated to assess the effect of the variation in the radiation pattern due to the metal plane. The size of the metal plane used in this work is $800 \times 800 \mathrm{~mm}^{2}$, which is electrically large compared to the antenna. Fig. 5 shows three different

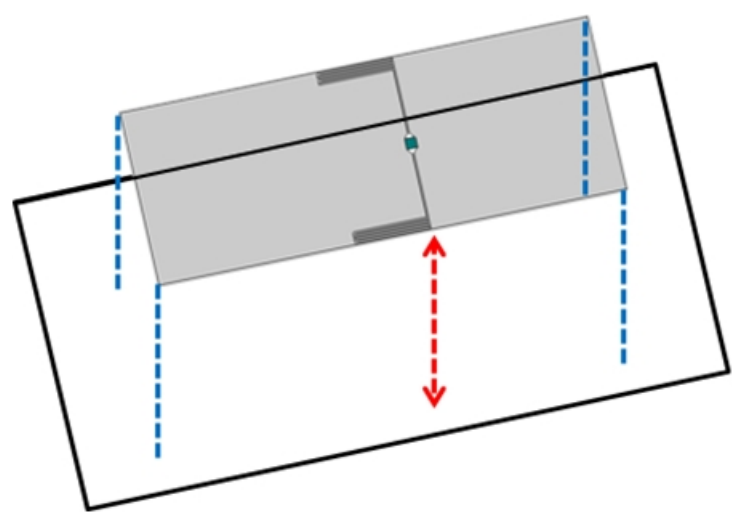

(a) Case 1

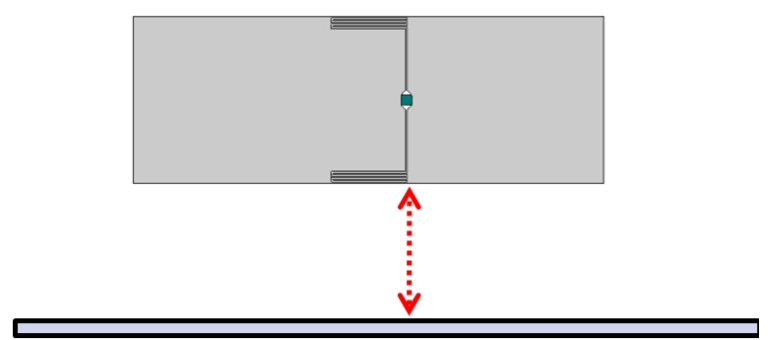

(b) Case 2

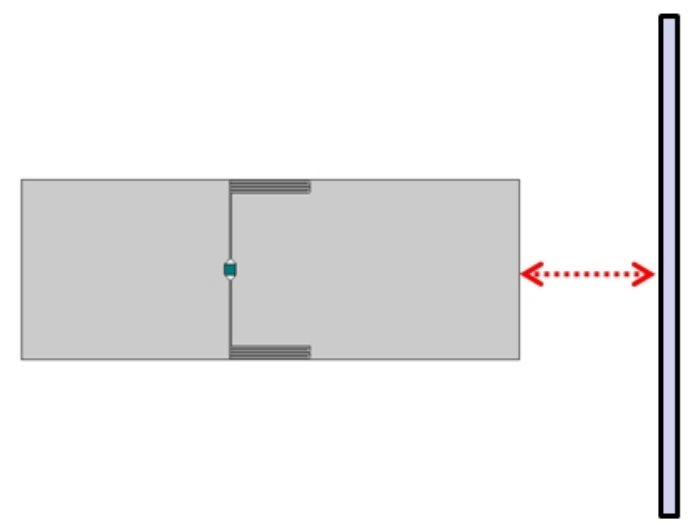

(c) Case 3

Fig. 5. Experiment configurations for mutual impedance and radiation pattern variation for the test antenna due to an adjacent metal plane. 
metal plane configurations where the metal plane is separated from the AUT by distance $d$. These are chosen to take into account most of the possible scenarios in which conductive materials would be placed near the tag antenna. In cases 1 and 2, the metal plane is located in the main lobe of the tag antenna, while in case 3 the metal plane is in the null direction. In particular, case 1 is important since it is directly related to the application of a metal tag antenna.

\section{3-2 Input Impedance Variation Due to Metal Plane}

Compared to conventional antennas for purposes other than mobile applications, impedance variation due to mutual coupling caused by nearby objects is a significant problem in RFID antennas. The amount of mutual coupling is negligible if a conductive material is located more than a half-wave length away.

As illustrated in the previous section, the input impedance of AUT located at various distances from a metal plane is investigated to identify the effect of the mutual coupling on the RFID performance. The input impedance is measured with the test fixture proposed in [19] in conjunction with the vector network analyzer. Fig. 6 shows the measured input impedances as a function of distance between the antenna and the metal plane. As expected, the amount of mutual coupling is different for different arrangements due to the unequal induced current distribution on the metal plane. The induced surface current is most significant when the metal plane is located in the main lobe and faced with AUT. Fig. 6(a) shows the input impedance of the AUT that changes more dramatically compared to other cases since the planar metallic plane is located in the main lobe region and faced with AUT. Fig. 6(b) shows a variation in the input impedance when the metallic plane is placed in the horizontal direction of the AUT. As expected, the antenna impedance changes less drastically than case 1 as expected. In case 3, the metal plane is located in the null direction of the AUT, revealing the least variation of the input impedance. The input resistance and the reactance increase sharply when the distance is small since the induced image source is in the same direction in contrast to cases 1 and 2. As discussed in the previous section, the increased mutual impedance results in matching degradation. In overall RFID systems, this matching degradation can be expressed with $\tau$, PTC (the Power Transmission Constant), which is defined as the delivered power and the available power. The reflection coefficient is determined with equation (2), and the PTC is derived from the reflection coefficient with equation (3).

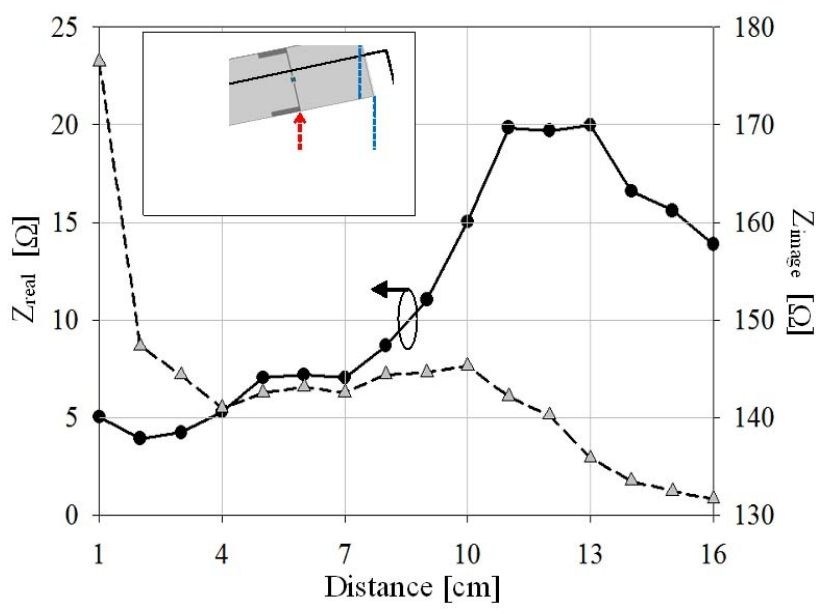

(a) Case 1

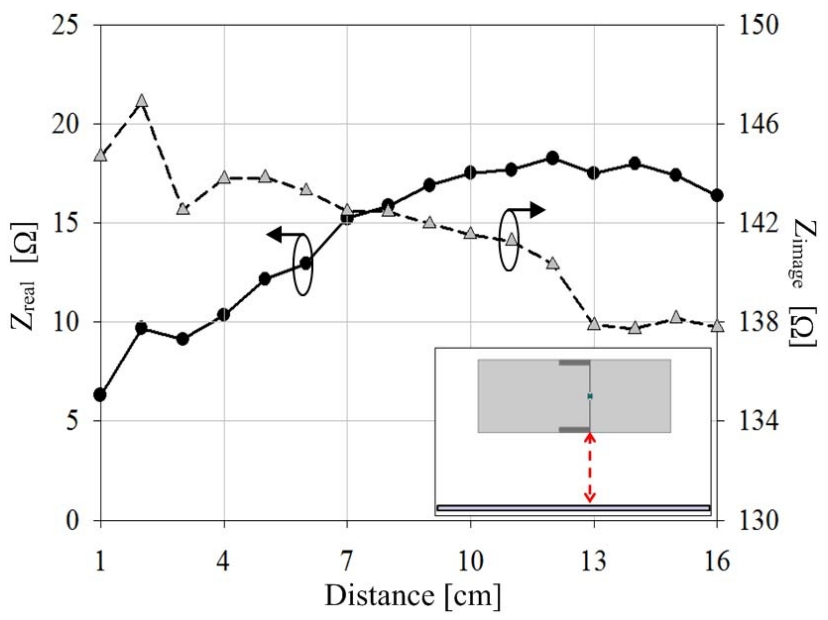

(b) Case 2

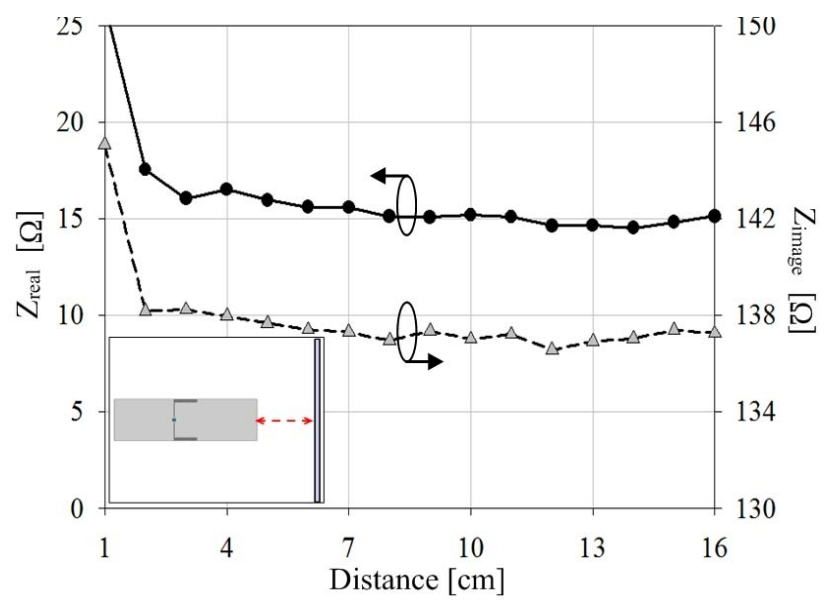

(c) Case 3

Fig. 6. Three measured input impedance variance profiles due to the adjacent metal plane.

$$
\begin{aligned}
& \Gamma=\frac{Z_{\text {chip }}-Z_{\text {ant }}^{*}}{Z_{\text {chip }}+Z_{\text {ant }}} \\
& \tau=1-|\Gamma|^{2}
\end{aligned}
$$




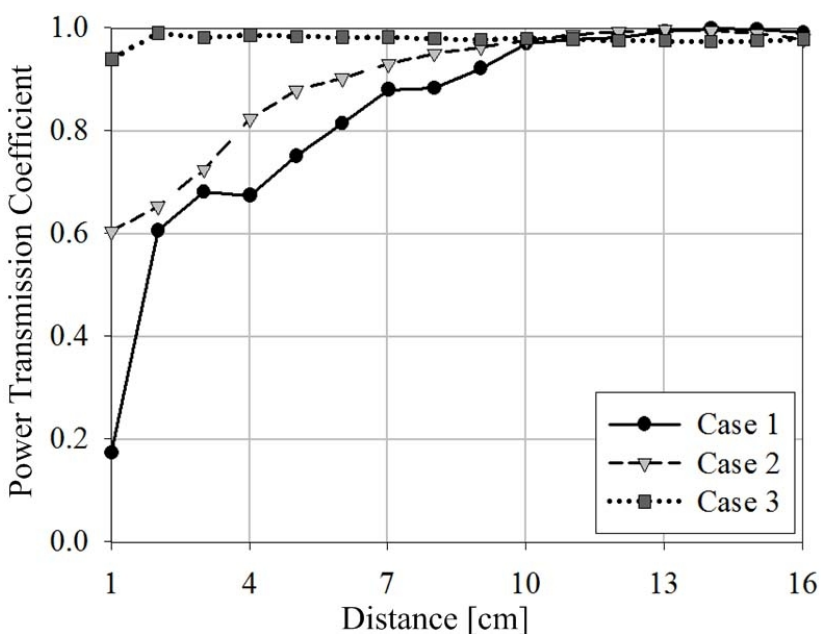

Fig. 7. The PTC variation of each case due to induced mutual impedance on the AUT.

The ratio of the power transfer between the tag chip and the tag antenna can be explained directly with the PTC, and the result is plotted in Fig. 7. As expected, the PTC at $16 \mathrm{~cm}$ is very close to 1 , which implies the maximum power transfer from the RFID chip to the antenna. However, the PTC gradually decreases as the distance decreases due to the increased mutual impedance of the antenna. The PTC behaves differently for different metal-AUT configurations, and case 1 reveals the most widely varying PTC due to its face-to-face arrangement.

\section{3-3 Gain Variation Due to the Metal Plane}

The input impedance and the radiation pattern are affected by a nearby metal plane. The metal plane effectively produces the image radiation current source behind the metal plane. Therefore, the radiation pattern of the AUT with the adjacent metal plane can be seen as an array antenna. The radiation pattern of a two-element array is theoretically composed with opposite-directed current source in cases 1 and 2, but the same direction for case 3. In general, 2-antenna array configurations can be divided into 3 cases according to the direction of the array axes, which are the x-, y-, and z-directions. The array factor for these 3 cases can be derived using the image theory.

$$
\begin{aligned}
& A F_{1}=2 j \sin [k h \sin (\theta) * \cos (\phi)] \\
& A F_{2}=2 j \sin [k h \sin (\theta) * \sin (\phi)] \\
& A F_{3}=2 \cos [k h \cos (\theta)]
\end{aligned}
$$

Furthermore, the element pattern is chosen as an infinitesimal dipole antenna because the radiation pattern

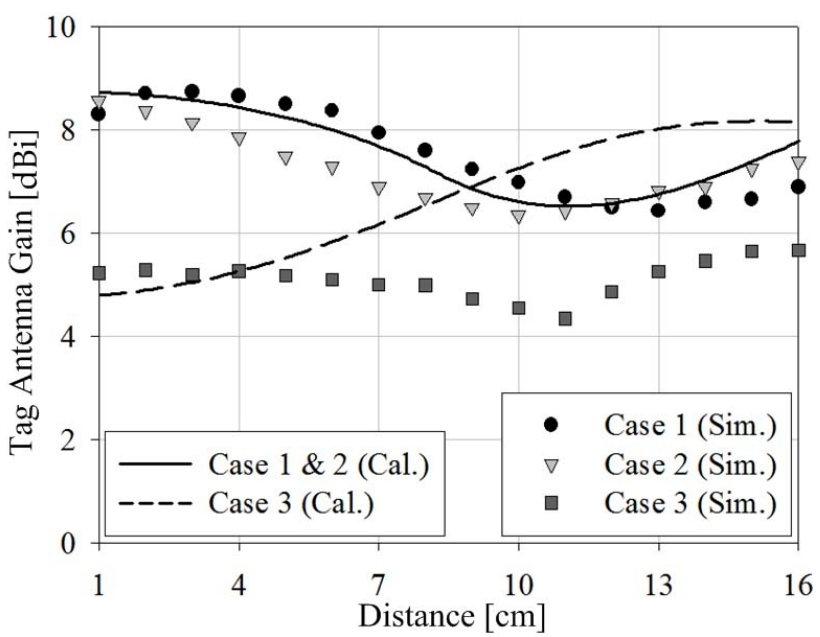

Fig. 8. Calculated and simulated antenna gain variations profiles due to the metal plane in 3 cases.

of a slot antenna array can be modeled with a dipole antenna by Babinet's principle [20]:

$$
E F=\sin (\theta)
$$

With the derived factors, the overall antenna gain of each case is estimated based on the assumption of an infinite ground plane. Fig. 8 shows the gain variation profiles. Cases 1 and 2 reveal that the gain of the test antenna increases greater than $8 \mathrm{dBi}$ and fluctuates as the distance varies. Case 3 shows a relatively small variation compared to cases 1 and 2; note that the simulation result deviates from the estimated result as the distance increases. The metal plane in the simulation is finite [21], [22], while the calculation assumes an infinite PEC ground plane so that the back lobe cannot propagate through the ground plane.

The radiation patterns in cases 1 and 2 are similar, and the antenna gains are precisely predicted. In contrast, the antenna gain for case 3 is less than $6 \mathrm{dBi}$, which is significantly different from the straight forward array theory derived from the image of the infinitesimal dipole. The vertical dipole above the imperfect finitesize ground plane causes the large back side lobe.

\section{Read Range Analysis}

In RFID systems or any system using the back-scattering mechanism, transmission performance can be categorized in terms of sensitivity characteristics. That is, if the back-scattered power from the tag is the bottleneck of the overall system performance, the transmission distance can be defined by equation (6). However, if the RFID tag chip is the bottleneck of the overall system performance, the reading range can be defined as $R_{2}$ : 


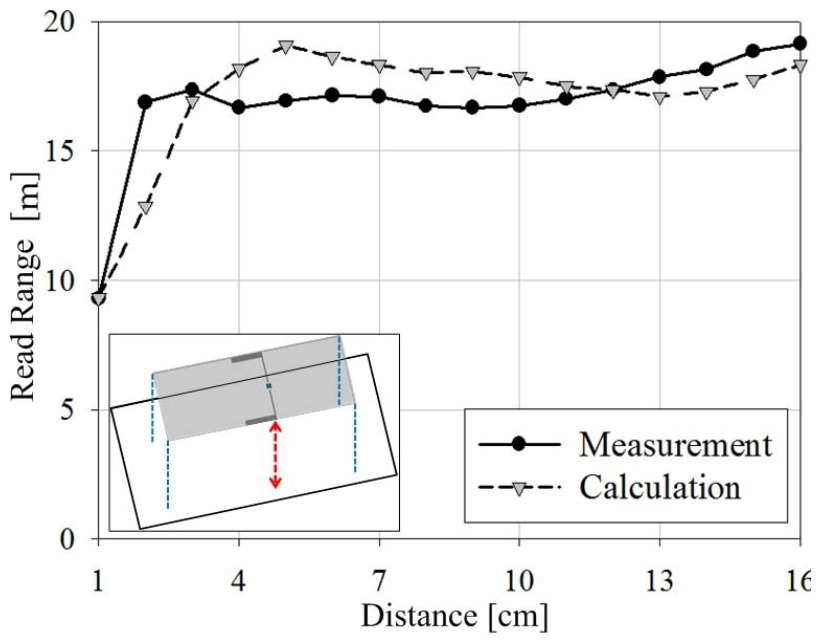

(a) Case 1

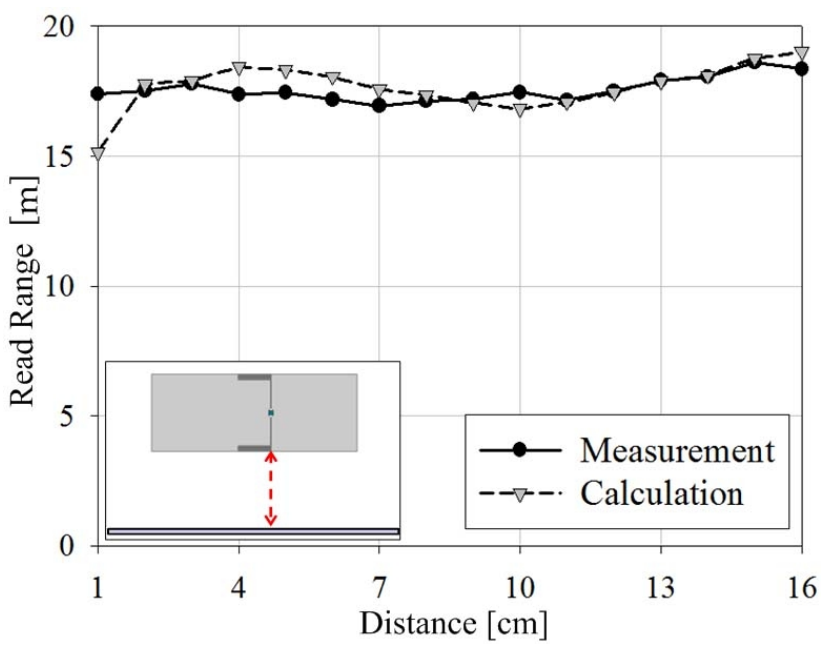

(b) Case 2

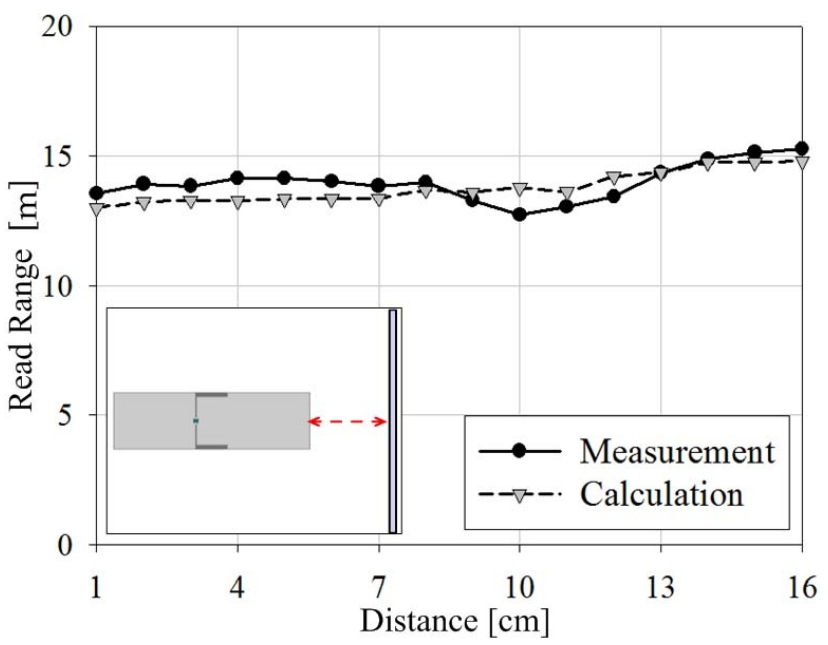

(c) Case 3

Fig. 9. Measured and calculated read ranges of 3 cases in the anechoic chamber.

$$
R_{1}=\frac{\lambda_{0}}{4 \pi} \sqrt{G_{\text {reader }} G_{\text {tag }} \tau} \sqrt[4]{\frac{P_{\mathrm{T}}}{P_{\text {min }}}}
$$

$$
R_{2}=\frac{\lambda_{0}}{4 \pi} \sqrt{G_{\text {reader }} G_{\text {tag }} \tau \frac{P_{\mathrm{T}}}{P_{\text {min }}}}
$$

where $\lambda_{0}$ is the wavelength at $912 \mathrm{MHz} \tau$ is the PTC of the antenna; $G_{\text {reader }}$ and $G_{\text {tag }}$ are the gain of the reader and the tag antennas, respectively; $P_{T}$ is the standard transmission power at the reader; and $P_{\min }$ is the minimum transmission power to activate the tag at the reader at a distance of $1 \mathrm{~m}$.

In realistic circumstances, the transceiver performance of a RFID tag chip is significantly inferior to a transceiver in a reader because the RFID tag chip has stringent size and performance limitations compared to the RFID reader system. That is why equation (7) is applicable in a real-world environment, and $R_{2}$ is chosen for transmission analysis in this work. There are 3 critical control variables, and they are the tag gain, the PTC, and the minimum required power at the tag antenna. An estimate of the read range can be obtained with controllable parameters. However, equation (7) cannot be applied when we measure the read range since these parameters are not measurable with conventional antenna measurement equipment. Measurement ports are not implemented in the tag, and moreover, the tag chip is directly connected to the antenna.

To measure the read range in an anechoic chamber environment, equation (7) should be modified to be represented with measurable quantities from the reader. One measurable variable is the sensitivity of the antenna at the reader side. By measuring the minimum power for the response of the tag at the reader side, the sensitivity can be derived with following equations [23]:

$$
\begin{aligned}
& S_{\text {tag }}=\frac{P_{\text {min_tag }}}{G_{\text {tag }} \tau}=\frac{P_{\text {min_reader }_{\text {rath }}}}{L_{\text {path }}} \\
& R=\frac{\lambda}{4 \pi} \sqrt{\frac{P_{T} G_{\text {reader }}}{S_{\text {tag }}}}=\frac{\lambda}{4 \pi} \sqrt{\frac{P_{T} G_{\text {reader }} L_{\text {path }}}{P_{\text {min_reader }}}}
\end{aligned}
$$

where $S_{\text {tag }}$ is the sensitivity of the tag antenna, $R$ is the measured read range, $L_{\text {path }}$ in equations (8) and (9) is the calibrated path loss at $1-\mathrm{m}$ distance between the tag and the reader, $P_{T}$ is $30 \mathrm{dBm}$, and $G_{\text {reader }}$ is $6 \mathrm{dBi}$ with linear polarization, so the EIRP is $36 \mathrm{dBm}$ in this study.

With the derived parameters, such as the PTC and the realized gain, the read ranges for thes 3 cases are calculated with equation (7) to predict the effect of a metal plane on tag performance. The estimated and measured read ranges are plotted in Fig. 9, and the estimated and measured results agree very well. Fig. 9 shows that read ranges for cases 1 and 2 of about $18 \mathrm{~m}$ and even $19 \mathrm{~m}$ are obtained for a 4-cm distance because the gain of the tag antenna increases over $8 \mathrm{dBi}$. Note that strong mu- 
tual coupling between the antenna and the metal plane renders a lower PTC, resulting in a decreased read range.

These 3 cases reveal distinctive matching degradation characteristics with different metal plane configurations. Case 1 suffers significant matching degradation, resulting in a degraded PTC as the metal plane is $1 \mathrm{~cm}$ away, and the read range decreases to $9.3 \mathrm{~m}$ from $19.1 \mathrm{~m}$. The PTC in case 2 decreased to 0.6 from 1, so the read range does not decrease as much as in case 1. Case 3 has a stable PTC, which is all more than 0.9 , so the read range lies between only $13 \mathrm{~m}$ and $14.8 \mathrm{~m}$.

\section{Conclusion}

The effects of an adjacent metal plane on RFID performances are derived and analyzed as a function of the degree of matching characteristics and gain variation. The most relevant 3 metal configurations are chosen to model the meandered slot RFID tag antenna in a real environment.

Cases 1 and 2 suffer matching degradation due to mutual impedance caused by electromagnetic coupling between the tag antenna and the metal plane face-to-face, so image radiation sources have an inverse direction that decreases the radiation resistance. Case 3 shows better matching characteristics, but the antenna gain enhancement is lower than in cases 1 and 2 because back lobe radiation occurred since the metal plane is located in the null direction of the original radiation pattern.

With the derived factors, the read range of the RFID tag system is predicted theoretically based on a modified Friis transmission equation. The read range is measured to confirm the validity of the prediction, and the results match the predicted values.

This research was supported by the MKE (The Ministry of Knowledge Economy), Korea, under the ITRC (Information Technology Research Center) support program supervised by the NIPA (National IT Industry Promotion Agency)" (NIPA-2012-H0301-121001).

\section{References}

[1] K. Seemann, F. Cielek, M. Schmidt, and R. Weigel, "RFID at UHF frequencies: The passive transponder fronted approach," Int. Conference on MIKON, pp. 657-661, May 2006.

[2] P. Foster, R. Burberry, "Antenna problems in RFID systems," IEEE Colloquium on RFID Technology,
London, UK, pp. 3/1-3/5, 1999.

[3] S. -L. Chen, K. -H. Lin, and R. Mittra, "Miniature and near-3D omnidirectional radiation pattern RFID tag antenna design," Electron. Lett., vol. 45, no. 18, pp. 923-924, Aug. 2009.

[4] J. Tan, X. Li, "Wideband double-UT RFID tag antenna design," IEEE Asia Pacific Conference on Circuits and Systems, Macao, pp. 1256-1259, 2008.

[5] C. Cho, H. Choo, and I. Park, "Broadband RFID tag antenna with quasi-isotropic radiation pattern," Electron. Letters., vol. 41, no. 20, pp. 1091-1092, Sep. 2005.

[6] Li Xu, Li-bin Tian, and Bin-jie Hu, "A novel broadband UHF RFID tag antenna mountable on metallic surface," IEEE J. Quantum Electron., pp. 2128-2131, 2007.

[7] H. -W. Son, C. -S. Pyo, "Design of RFID tag antennas using an inductively coupled feed," Electron. Letters, vol. 41, no. 18, pp. 994-996, Sep. 2005.

[8] W. Choi, H. -W. Son, J. -H. Bae, and G. Choi, "RFID tag antenna with a meandered dipole and inductively coupled feed," IEEE AP-S. Int. Symp., pp. 619-622, Jul. 2006.

[9] S. -H Lim, Y. -C Oh, H. Lim, and N. -H. Myung, "Compact wideband tag antenna for UHF RFID," Microwave and Optical Technology Letters, vol. 51, no. 5, pp. 1291-1294, May 2009.

[10] G. Marrocco, "RFID antennas for the UHF remote monitoring of human subjects," IEEE Trans. Antennas and Propagation, vol. 55, no. 6, pp. 18621870, Jul. 2007.

[11] J. Kim, I. -Y. Oh, T. -W. Koo, J. -C. Kim, D. Kim, and J. -G. Yook, "Design of a meandered slot antenna for the UHF RFID application," IEEE AP-S. Int. Symp., pp. 1-4, Jul. 2010.

[12] L. Ukkonen, L. Sydänheimo, and M. Kivikoski, "Effects of metallic plate size on the performance of microstrip patch type tag antennas for passive RFID," IEEE Antennas and Wireless Propagation Letters, vol. 4, pp. 410-413, Oct. 2005.

[13] L. Mo, H. Zhang, "RFID antenna near the surface of metal," Int. Symp. on Microwave, Antenna, Propagation, and EMC Technologies for Wireless Communications, Hangzhou, pp. 803-806, 2007.

[14] X. Qing, Z. N. Chen, "Proximity effects of metallic environments on high frequency RFID reader antenna: Study and applications," IEEE Trans. on Antennas and Propagation, vol. 55, no. 11, pp. 31053111, Nov. 2007.

[15] D. M. Dobkin, S. M Weigand, "Environmental effects on RFID tag antennas," Microwave Symposium Digest, 2005 IEEE MTT-S International, Jun. 
2005.

[16] M. Keskilammi, L. Sydänheimo, and M. Kivikoski, "Radio frequency technology for automated manufacturing and logistics control. Part 1: Passive RFID systems and the effects of antenna parameters on operational distance," The Int. J. of Advanced Manufacturing Technology, vol. 21, no. 10-11, pp. 769-774, 2003.

[17] T. -W. Koo, D. Kim, J. -I. Ryu, J. -K. Kim, J. -G. Yook, and J. -C. Kim, "Design and implementation of label-type UHF RFID tags for the metallic object application," IEEE AP-S. Int. Symp., pp. 1-4, Jul. 2010.

[18] J. T. Prothro, G. D. Durgin, and J. D. Griffin, "The effects of a metal ground plane on RFID tag antennas," Antennas and Propagation Society International Symposium 2006, IEEE, pp. 3241-3244, Jul. 2006.

[19] D. Kim, T. -W. Koo, J. -I. Ryu, J. -G. Yook, and

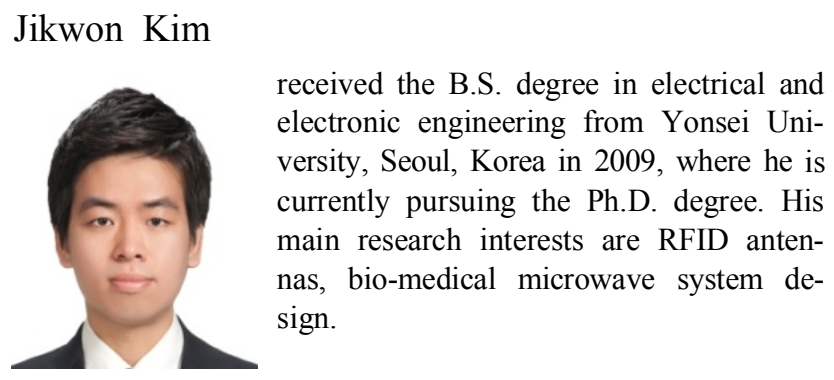

\section{Il-Young Oh}

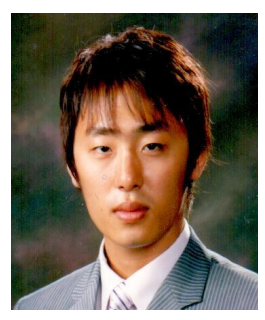

received the B.S. degree in electrical and electronic engineering from Yonsei University, Seoul, Korea in 2007, where he is currently pursuing the Ph.D. degree. His main research interests are numerical analysis based on low dispersion finitedifference time-domain (FDTD) method, plasma analysis, and HEMP coupling.
J. -C. Kim, "Accurate impedance measurement and implementation of a folded dipole antenna for RFID tag," IEEE AP-S Int. Symp. Antenna and Propagation, pp. 93-96, 2009.

[20] R. Elliott, L. Kurtz, "The design of small slot arrays," Antennas and Propagation, IEEE Transactions on, vol. 26, no. 2, pp. 214-219, Mar. 1978.

[21] X.-B. Xu, Y. Huang, "An efficient analysis of vertical dipole antennas above a lossy half-space," Progress in Electromagnetics Research, vol. 74, pp. 353-377, 2007.

[22] K. Sarabandi, M. D. Casciato, and I. S. Koh, "Efficient calculation of the fields of a dipole radiating above an impedance surface," Antennas and Propagation, IEEE Transactions on, vol. 50, no. 9, pp. 1222-1235, Sep. 2002.

[23] P. V. Nikitin, K. V. S. Rao, "Antennas and propagation in UHF RFID systems," 2008 IEEE Int. Conference on RFID, pp. 277-288, Apr. 2008.

Tae-Wan Koo

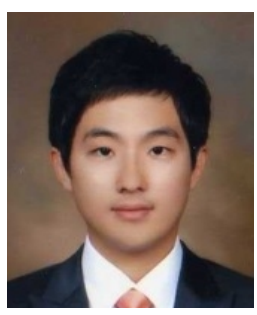

received the B.S. degree in electronic engineering from Hankuk University of Foreign Studies, Gyeonggido, Korea, in 2009, and M.S. degree in electrical and electronic from Yonsei University, Seoul, Korea. He is currently pursuing the Ph.D. degree in electrical and electronic engineering from Yonsei University, Seoul, Korea. His research interests are design and implementation of wide-band/multi-band antennas for wireless communication systems.

Jun Chul Kim

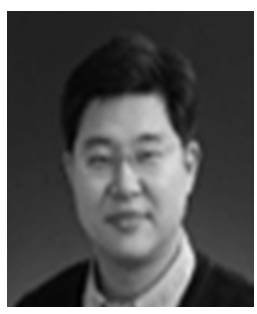

received the B.S. degrees in Physics from the Sogang University in 1991, and the M.S. and the Ph.D degrees in electronic engineering from the Sogang University, Seoul, Korea, in 1993 and in 1998, respectively. In 1996, has joined KETI, Korea Electronics Technology Institute, where he has performed the research on the Duplexer and Filter for mobile communications. His current research interests include the design components \& modules using embedded passives. 


\section{Dongsu Kim}

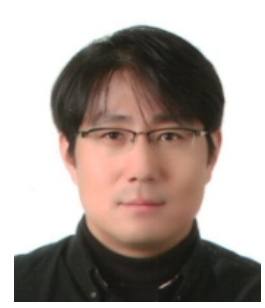

received B.S. and M.S. degrees in electronic and electrical engineering from $\mathrm{Ky}$ ungpook National University, Daegu, Korea, in 1997 and 1999, respectively, and M.S. and Ph.D. degrees in electrical and computer engineering from the Georgia Institute of Technology, Atlanta, USA, in 2001 and 2004, respectively. His doctoral work included design, fabrication, and characterization of ferroelectric devices and beamforming networks for smart antenna systems. In 2001, he worked for MicroCoating Technologies, Chamblee, GA, where he was involved with design and analysis of ferroelectric devices. In 2004, he joined the Packaging Research Center of Korea Electronics Technology Institute in Korea. His research interests include ferroelectric tunable devices, LTCC-, PCB-, Si-based multilayer modules, GaN-based power amplifiers, and system-in-package.

\section{Jong-Gwan Yook}

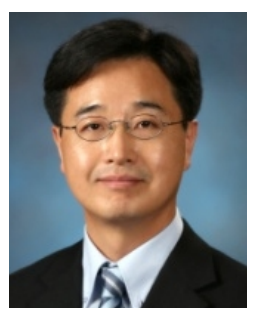

(S'89 M'97) was born in Seoul, Korea. He received the B.S. and M.S. degrees in electronics engineering from Yonsei University, Seoul, Korea, in 1987 and 1989, respectively, and the Ph.D. degree from the University of Michigan, Ann Arbor, MI, in 1996.

He is currently a Professor with the School of Electrical and Electronic Engineering, Yonsei University. $\mathrm{He}$ is also serving IEEE EMC Society as a Distinguished Lecturer in the year 2012 to 2013 . His main research interests are in the areas of theoretical/numerical electromagnetic modeling and characterization of microwave/millimeterwave circuits and components, design of radio frequency integrated circuits (RFIC) and monolithic microwave integratedcircui t (MMIC), and analysis and optimization of high-frequency high-speed interconnects, including signal/power integrity (EMI/EMC), based on frequency as well as time-domain full-wave methods. Recently, his research team is developing various biosensors, such as carbon nano-tube RF biosensor for nanometer size antigen-antibody detection as well as remote wireless vital signal monitoring sensors. 